\title{
Tilt anisoplanatism in laser-guide-star-based multiconjugate adaptive optics
}

\section{Reconstruction of the long exposure point spread function from control loop data}

\author{
R. C. Flicker ${ }^{1,2}$, F. J. Rigaut ${ }^{2}$, and B. L. Ellerbroek ${ }^{2}$ \\ 1 Lund Observatory, Box 43, 22100 Lund, Sweden \\ 2 Gemini Observatory, 670 N. A'Ohoku Pl., Hilo HI-96720, USA
}

Received 22 August 2002 / Accepted 6 January 2003

\begin{abstract}
A method is presented for estimating the long exposure point spread function (PSF) degradation due to tilt anisoplanatism in a laser-guide-star-based multiconjugate adaptive optics systems from control loop data. The algorithm is tested in numerical Monte Carlo simulations of the separately driven low-order null-mode system, and is shown to be robust and accurate with less than $10 \%$ relative error in both $H$ and $K$ bands down to a natural guide star (NGS) magnitude of $m_{R}=21$, for a symmetric asterism with three NGS on a $30 \operatorname{arcsec}$ radius. The $H$ band limiting magnitude of the null-mode system due to NGS signal-to-noise ratio and servo-lag was estimated previously to $m_{R}=19$. At this magnitude, the relative errors in the reconstructed PSF and Strehl are here found to be less than 5\% and 1\%, suggesting that the PSF retrieval algorithm will be applicable and reliable for the full range of operating conditions of the null-mode system.
\end{abstract}

Key words. Instrumentation - adaptive optics - methods - statistical

\section{Introduction}

\subsection{Multiconjugation and laser guide stars}

The potential benefits of conventional adaptive optics (AO) for astronomy are being limited by two severe shortcomings: low sky coverage and strong angular anisoplanatism. The sky coverage is the fraction of the sky within which AO provides a useful level of turbulence compensation. For conventional adaptive optics instruments (e.g. PUEO, Hokupa'a, NACO) this is commonly only a few percent (Rigaut \& Gendron 1992; Ellerbroek \& Tyler 1998) due to the necessity of having a bright natural guide star (NGS) close to the science target. A low sky coverage greatly reduces the scientific return of an adaptive optics system and limits its use to observation programs which can supply the reference star. Anisoplanatism, secondly, manifests as a field-varying point spread function (PSF) and a loss of turbulence compensation performance when the correlation between the line-of-sight turbulence and the measured column turbulence decreases. Angular anisoplanatism arises when observing off-axis from the wavefront reference source, and focal anisoplanatism arises in addition when the reference source is situated at a finite distance. A strongly varying PSF complicates post-processing of the image, and without precise knowledge of the PSF, the accuracy of photometry and deconvolution

$\overline{\text { Send offprint requests to: } \mathrm{R}}$. C. Flicker, e-mail: ralf@astro.lu.se algorithms is much reduced. Set to amend the two failings, to increase the sky coverage and reduce anisoplanatism, are the emerging technologies of, respectively, laser guide stars (LGSs) and multi-conjugated adaptive optics (MCAO).

Recent years have seen a maturing of LGS technology, and laser-based AO systems have now been successfully prototyped at several observatories, such as, e.g., Starfire Optical Range (Fugate 1994), Lick Observatory (Gavel \& Friedman 1998), Calar Alto Observatory (Hippler et al. 2000). A laser is launched from a small telescope near the observing telescope, and the backscattered light is used by the wavefront sensor (WFS) in the adaptive optics instrument to measure the atmospheric aberrations within the cone traced out by the LGS. Atmospheric tilt jitter on the uplink will cause the laser spot to wander around on the sky, however, and as first pointed out by Pilkington et al. (1987) since the scattered photons received in the WFS will have traversed some of the same tilt mode, the signal is partially canceled. Hence, the global tip and tilt can not be inferred from the LGS measurement itself due to the low signal-to-noise ratio $(S / N)$, and one must employ an auxiliary NGS to obtain independent tip/tilt measurements. This would seem to reintroduce the sky coverage problem one set out to solve with laser guide stars in the first place, but calculations show that the use of the entire telescope to collect photons and running the NGS servo at a lower bandwidth allows for a fainter NGS limiting magnitude, and thus a reasonable sky 


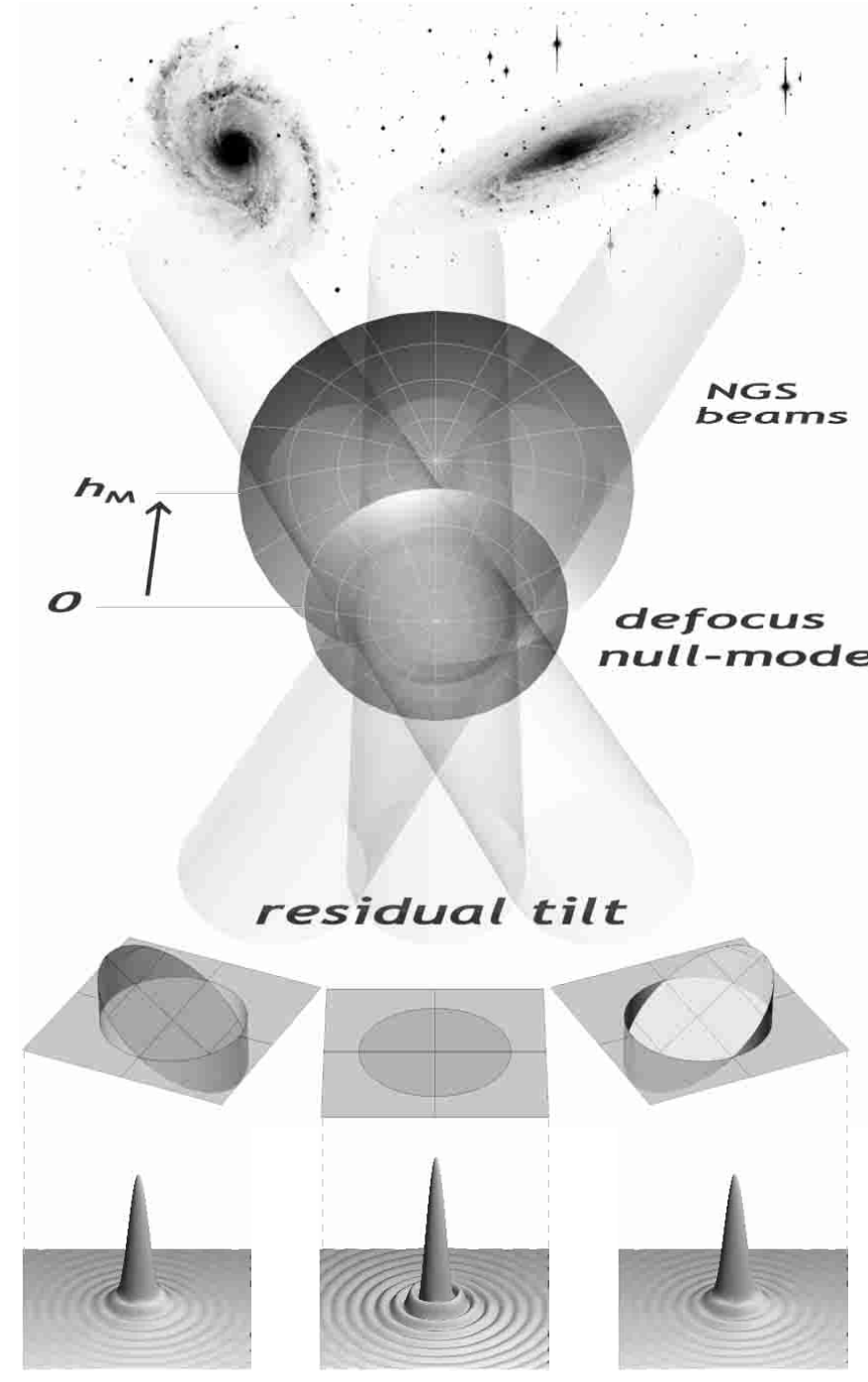

Fig. 1. Example of a tomographic null-mode for LGSs: a focus mode at an altitude $h_{\mathrm{M}}$ is canceled on-axis by a correction in the pupil plane, which leaves a differential tilt mode uncorrected off-axis. The PSFs at the bottom (square root scaling) are taken from the sample configuration presented in Sect. 3, and show the realistic loss of contrast and Strehl ratio in a long exposure due to residual null-mode aberrations. The on-axis Strehl ratio is 0.93 , which has dropped to 0.74 at $50^{\prime \prime}$ off-axis.

coverage (Rigaut \& Gendron 1992; Parenti \& Sasiela 1994). Although the most viable technique at the present, alternatives have been proposed which may come to solve the LGS tip/tilt determination problem in a near future; most notably polychromatic LGSs that exploit atmospheric dispersion to retrieve the tip/tilt mode (see Foy et al. 2000).

To address the second shortcoming of conventional AO, anisoplanatism, the concepts of atmospheric tomography and multi-conjugation were introduced to the adaptive optics community in the late 1980s (Beckers 1988). Site surveys at various astronomical sites have revealed turbulence profiles that are characterized by a small number of thin layers within which most of the turbulence is being generated (Racine \& Ellerbroek 1995; Vernin et al. 2000). It has been shown that anisoplanatism would be mitigated and angular coherence increased if one could optically conjugate the deformable mirror (DM) to where the turbulence is the strongest (Fried 1992). Employing multiple DMs, one can envision dedicating one DM to each prominent turbulence layer. Such multi-conjugation increases the number of degrees of freedom of an AO system dramatically, introducing the vertical dimension. Assigning sensible shapes to multiple DMs will require multiple wavefront measurements over the field of view, covering the the entire volume of atmosphere interfering with the science target. It then becomes the task of a tomographic reconstruction algorithm to combine the information received from all the guide stars in order to resolve the vertical structure of the turbulence, and project it onto the DMs conjugated to turbulence. Tomography in conjunction with multi-conjugation is now commonly referred to as MCAO, and it would enable one to adaptively correct turbulence-induced aberrations over a field of view rather than merely a single direction, potentially eliminating anisoplanatism completely within the field of view. An MCAO system of the type described above which relies on NGSs for wavefront sensing, however, will have a vanishingly small sky coverage, and an entirely new class of problems arises when we look to laser-guide-star-based MCAO.

\subsection{Null-modes and tilt anisoplanatism}

Due to the tip/tilt determination problem associated with LGSs, the tomographic wavefront reconstruction in MCAO will be impaired and unable to determine the altitudes of a subset of low-order modes. The classification of the problem depends upon the particular subspace of controlled modes, but referring to a Zernike basis allows a convenient description of the problem. As a consequence of the tilt filtering, the MCAO system looses all tomographic information about the altitudes of quadratic modes, i.e., one focus and two astigmatisms. Left to itself, the laser-based MCAO system would accurately measure the curvature, but lacking any information on the altitude of the mode it would conclude that it was all located at the ground, i.e. zero altitude, and consequently assign all correction to the pupil-conjugated DM. As a result, the mode will be perfectly corrected on-axis, but off-axis only the quadratic parts will cancel and leave a field-dependent tilt component uncorrected. One may easily convince oneself of this by inspecting the off-axis residual of a focus mode $Z_{4}$ at an altitude $h$ which is corrected on the ground by $-Z_{4}$ (cf. Fig. 1). At an off-axis angle $\alpha$ in the $x$-direction, the residual mode is $Z_{4}(x+h \alpha, y)-Z_{4}(x, y)=2 \sqrt{3}\left(2 x h \alpha+h^{2} \alpha^{2}\right)$, which is proportional to Zernike tilt, $Z_{2}=2 x$, and an additional piston term. If this was a WFS measurement, $\alpha$ is given by the position of the guide star, but since the WFS is insensitive to piston, the altitude $h$ of the upper focus mode can only be inferred from the tilt term. For a laser guide star ${ }^{1}$, this term is filtered, and the measurement returns null. Failure to correct this differential tilt will result in differential image motion, or tilt anisoplanatism,

\footnotetext{
${ }^{1}$ Due to the cone effect, LGSs will be blind to combinations of quadratic modes of slightly different amplitudes, as the pupil of the LGS cone at a non-zero altitude is smaller than the telescope pupil.
} 
which will manifest in an MCAO image as a convolution of the long exposure PSF with a field-varying Gaussian.

The problem of tilt anisoplanatism in LGS-based multiconjugate adaptive optics was observed and investigated already in 1995 (Fried 1995), but only recently addressed in an engineering context (Ellerbroek \& Rigaut 2001), with the advent of MCAO systems currently being designed (e.g. Gemini-South Adaptive Optics). We shall refer to the set of modes that can not be inferred from LGS measurements as null-modes, since they belong to the null space of the LGS DM-to-WFS interaction matrix. In addition to the global tip and tilt modes which are null to a single laser guide star, three null-modes consisting of paired quadratic modes that cancel on-axis arise in the context of MCAO with LGSs, and they shall require special treatment. Looking to a natural guide star solution to measuring the null-modes and omitting a discussion of possible LGS scenarios, two basic options present themselves: (a) a single NGS and WFS measuring the five first Zernikes, or (b) a minimum of three NGSs and WFS measuring only tip and tilt over the field. For the GSAO system one has opted for alternative (b), and attention will be restricted to this method in this paper; alternative (a) has been investigated to some extent in (Le Louarn 2001). Assuming the high-order turbulence compensation achieved by the LGS system to provide a high and uniform Strehl ratio over the field of view, most of the resultant anisoplanatism may plausibly result from residual null-modes. Previous studies have investigated the parameter space of nullmode compensation and discussed performance optimization (Ellerbroek \& Rigaut 2001; Flicker \& Rigaut 2001). The aim of this paper is to derive an algorithm for real-time estimation of the tilt anisoplanatism PSF using available control loop data.

\subsection{Point spread function estimation algorithm}

It is important to recognize that the algorithm to be presented does not in any way purport to obviate existing conventional PSF extraction and photometry techniques, and that the tilt anisoplanatism PSF is only one in a series that must be convolved together to give the resultant PSF of a LGS-based MCAO system. For one thing, by deriving these types of algorithms for MCAO now and hardwiring them into the real-time data processing architecture, the PSF estimation will come for free along with the observation at no cost at all to the observer once the instrument gets built. The ultimate goal is of course to be able to present the observer with a very accurate systemderived estimation of the image PSF over the field of view that requires virtually no post-processing at all on the part of the astronomer. The role of image-independent PSF estimation algorithms in adaptive optics has not yet been entirely defined or explored, as it concerns a relatively recent development within astronomical instrumentation. But it is clear that they will be an integral part of future MCAO systems - partly because it is possible, and partly because they will sometimes provide a valuable tool not available through any other means. Where aperture photometry on isolated stars is the sole interest, the algorithm is of little use to the astronomer, but two obvious examples of where it can make a difference are photometry on crowded star fields and deconvolution of extended objects. Obtaining an accurate PSF in these two cases when the PSF is varying strongly and in a nonlinear way is notoriously difficult, and conventional post-processing methods are likely to fall short or produce a very rough approximation of the PSF. It is clear, however, that MCAO images will always contain some degree of anisoplanatism even in the best of cases, and so it becomes the second most important task to identify and map these variations over the field if they can not be corrected for. But of course the utility of this type of algorithm goes beyond that of merely supporting PSF photometry and deconvolution. Apart from PSF reconstruction, the algorithm provides a tool for engineers to diagnose the system in real time in order to fine tune parameters, and also logging the performance in order to collect statistics that can help characterizing the atmospheric turbulence on the site.

Section 2 describes the system operation briefly and addresses how to obtain the quantities relevant for PSF estimation from loop data. In Sect. 3 the link to the estimated PSF from loop data is established, some sample results are given in Sect. 4, and a brief discussion offered in Sect. 5. It should be noted that variations on MCAO have been proposed that could circumvent the problematics associated with LGSs, as described above. Most notably, the concept of layer-oriented MCAO as developed by Ragazzoni (2000) promises to retain some sky coverage while dispensing with LGSs by instead combining the light from many NGSs over a large field of view. Layer-oriented MCAO obviates the need for null-mode compensation, and foregoes the concerns addressed in this paper.

\section{Null-mode system analysis}

The transfer-function-based analytical model for tilt anisoplanatism studied previously (Flicker \& Rigaut 2001) is a useful PSF prediction tool, but it turns out to be not easily adapted into a real-time or post-processing PSF retrieval algorithm. Here such an algorithm is derived which requires only knowledge of the NGS positions and magnitudes, and an estimate of the WFS noise covariance matrix (although it will be shown subsequently that precise knowledge of the latter is not required, and that the algorithm is very forgiving with respect to errors in this parameter). It will be assumed that the null-mode control system is completely decoupled from the high-order LGS system. This requires the LGS interaction matrix to be projected onto a subspace of controllable modes orthogonal to the nullmodes. If the decoupling is imperfect due to lack of orthogonality or injection of noise in the LGS system, this will only affect the relative share of quadratic mode compensation between the null-mode system and the high-order system. Since the PSF reconstruction algorithm to be presented is based upon the residual mode, i.e. the mode seen by the science camera, it is insensitive to how this mode was produced, which really does not matter other than from the point of view of modal control optimization. It shall also be assumed that the null-mode system is able to perfectly produce the three quadratic Zernike modes, i.e., no consideration is taken of the fitting of influence functions to Zernikes. This appears to be a reasonable assumption in light of the current trend of development of adaptive 
optics systems, tending toward very high-order systems with many hundred degrees of freedom. In a modal framework, the WFS and mode reconstruction equations may be written

$s(t)=B \boldsymbol{\alpha}(t)+m(t)$

$\widehat{\alpha}(t)=E s(t)$,

where $s$ the closed loop wavefront sensor measurement and $m$ the WFS noise, $\boldsymbol{\alpha}$ is the vector of residual mode coefficients and $\widehat{\alpha}$ its estimate, $B$ the mode-to-WFS interaction matrix and $E$ the modal reconstruction matrix. For an adequately resolved NGS asterism, the range of $B$ will be perfectly isomorph to the space spanned by the null-modes. This means that there is only the trivial null-space, and $E B=I$ is always verified. If it becomes necessary to observe with a poorly spatially resolved asterism or with very faint guide stars, a noise-weighted least-squares estimator or an optimal estimator can be shown to significantly improve performance (Flicker \& Rigaut 2001). The Gauss-Markov noise-weighted estimator $E=\left(B^{\mathrm{T}} C_{\mathrm{m}}^{-1} B\right)^{-1} B^{\mathrm{T}} C_{\mathrm{m}}^{-1}$, where $C_{\mathrm{m}}$ is the noise covariance matrix, still verifies $E B=I$, and the minimum variance estimator can be constrained to do so as well (Ellerbroek 1994) - estimators violating this constraint will not be considered in this study. Exploiting this constraint, the mode reconstruction equation becomes $\widehat{\alpha}=\alpha+n$, where $n=E m$ is the WFS noise propagated onto the null-modes. The noise-free residual mode $\alpha$ is the sum of the turbulent mode and the correction applied by the null-mode system. Hence, it is the quantity governing the formation of the long exposure point spread function, and its statistics in the form of the covariance matrix $C_{\alpha}$ will lead to the long-exposure PSF estimation.

\subsection{Residual mode statistics}

Noise in the LGS WFSs will propagate into a tomographic reconstruction error that can be thought of as either a noise on the modal content, or a noise on the tomography of correctly identified modes. Whichever way you turn that around, it is clear that some quadratic null-modes are going to be injected from the high-order system and left for the null-mode system to deal with. It is deemed, however, that this contribution will in most cases of interest be negligibly small due to the steep decrease of the Noll coefficients (Noll 1976) and the use of laser guide stars that are sufficiently bright that the noise injection will be small in any case. Hence, this small contribution will not be considered in the following analysis. Expanding the residual phase aberration $\varphi$ in the plane of the telescope pupil of radius $R=D / 2$ on the null-mode basis gives

$\varphi(\boldsymbol{x}, \boldsymbol{\theta}, t)=\sum_{j=2}^{6} \alpha_{j}(t) Z_{j}\left(\frac{\boldsymbol{x}}{R}\right)-\sum_{j=4}^{6} \beta_{j}(t) Z_{j}\left(\frac{\boldsymbol{x}+\boldsymbol{\theta} h_{\mathrm{M}}}{R_{\mathrm{M}}}\right)$

where $R_{\mathrm{M}}$ is the radius of the meta-pupil on the DM conjugated to the range $h_{\mathrm{M}}, \beta_{j}$ the mode coefficients of the conjugated DM (proportional to the $\alpha_{j}$ ), and the $Z_{j}$ are Zernike's circular polynomials. They are orthogonal and normalized to unit variance on the disk of unit radius $\Omega$ :

$\delta_{i j}=\int_{\Omega} \mathrm{d} \boldsymbol{x} w(\boldsymbol{x}) Z_{i}(\boldsymbol{x}) Z_{j}(\boldsymbol{x}), \quad w(\boldsymbol{x})= \begin{cases}\frac{1}{\pi}, & \boldsymbol{x} \in \Omega \\ 0, & \text { otherwise. }\end{cases}$

In Cartesian coordinates, the five first Zernikes (omitting piston, $j=1$ ) are

$\begin{array}{ll}Z_{2}=2 x, & Z_{4}=\sqrt{3}\left[2\left(x^{2}+y^{2}\right)-1\right], \\ Z_{3}=2 y, & Z_{5}=2 \sqrt{6} x y \\ & Z_{6}=\sqrt{6}\left(x^{2}-y^{2}\right) .\end{array}$

Substituting the Zernikes into (3) and imposing zero curvature on-axis by constraining the coefficients $\beta_{j}$ of the altitudeconjugated $\mathrm{DM}$ to be $\beta_{j}=\left(R_{\mathrm{M}} / R\right)^{2} \alpha_{j}$, gives the residual phase $\varphi$ as a linear combination of tilt errors:

$$
\begin{aligned}
\varphi(\boldsymbol{x}, \boldsymbol{\theta}, t)= & \frac{2}{R}\left(\alpha_{2} x+\alpha_{3} y\right)-\alpha_{4} \frac{4 \sqrt{3} h_{\mathrm{M}}}{R^{2}}\left(x \theta_{x}+y \theta_{y}\right) \\
& -\frac{2 \sqrt{6} h_{\mathrm{M}}}{R^{2}}\left[\alpha_{5}\left(y \theta_{x}+x \theta_{y}\right)-\alpha_{6}\left(x \theta_{x}-y \theta_{y}\right)\right] .
\end{aligned}
$$

Recombining coefficients, one may write this as

$\varphi(\boldsymbol{x}, \boldsymbol{\theta}, t)=\sum_{k=2}^{3} \gamma_{k}(\boldsymbol{\theta}, t) Z_{k}(\boldsymbol{x} / R)$,

where the field dependent tip/tilt coefficients $\gamma_{k}$ are given by

$\gamma_{k}(\boldsymbol{\theta}, t)=\sum_{i=2}^{6} \alpha_{i}(t) T_{k i}(\boldsymbol{\theta}), \quad k=2,3$

and the matrix $T(\boldsymbol{\theta})$ is

$T=\left[\begin{array}{lll}1 & 0 & -2 \sqrt{3} \frac{h_{\mathrm{M}}}{R} \theta_{x}-\sqrt{6} \frac{h_{\mathrm{M}}}{R} \theta_{y}-\sqrt{6} \frac{h_{\mathrm{M}}}{R} \theta_{x} \\ 0 & 1 & -2 \sqrt{3} \frac{h_{\mathrm{M}}}{R} \theta_{y}-\sqrt{6} \frac{h_{\mathrm{M}}}{R} \theta_{x}+\sqrt{6} \frac{h_{\mathrm{M}}}{R} \theta_{y}\end{array}\right]$.

The first two columns represent the contribution from global tip and tilt, and the remaining three represent the differential tilt arising from cancellation of quadratic modes. Establish the notation $C_{v} \equiv\left\langle v v^{\mathrm{T}}\right\rangle$ for the covariance matrix of a stochastic vector $v$, where angular brackets signify statistical averaging and superscript ${ }^{\mathrm{T}}$ the transpose operator. The residual nullmode phase variance is then

$\sigma_{\varphi}^{2}=\operatorname{tr}\left(C_{\gamma}\right)=C_{\gamma}^{22}+C_{\gamma}^{33}$

on account of the orthogonality of the Zernikes. The elements of the tilt covariance matrix $C_{\gamma}$ are

$C_{\gamma}^{k l}=\sum_{i=2}^{6} \sum_{j=2}^{6} T_{k i} C_{\alpha}^{i j} T_{l j}$

or simply $C_{\gamma}=T C_{\alpha} T^{\mathrm{T}}$ in a matrix notation. The residual tilt covariance matrix $C_{\gamma}$ was computed analytically in (Flicker \& Rigaut 2001), where atmospheric and noise statistics had to be known a priori. In Sect. 3 it will be shown how $C_{\gamma}$ relates to the PSF, and this section concludes by showing how it may be retained from available closed loop data produced by the system during the observation. Since the mode noise $n$ is not 
correlated to the simultaneous residual mode $\alpha$, we have that $C_{\alpha}=C_{\widehat{\alpha}}-C_{n}$, which upon substituting (2) reads $C_{\alpha}=E\left(C_{\mathrm{s}}-\right.$ $\left.C_{\mathrm{m}}\right) E^{\mathrm{T}}$, where $C_{\mathrm{s}}$ is the closed loop WFS covariance matrix and $C_{\mathrm{m}}$ the WFS noise covariance matrix. This gives the final expression

$C_{\gamma}=T E\left(C_{\mathrm{s}}-C_{\mathrm{m}}\right) E^{\mathrm{T}} T^{\mathrm{T}}$,

where $C_{\mathrm{s}}$ is readily obtained from loop data, and $C_{\mathrm{m}}$ may be computed analytically with adequate accuracy for the most common types of wavefront sensors. Robustness of the PSF estimation with respect to errors in $C_{\mathrm{m}}$ is investigated in Sect. 3, where it is shown that the estimate of $C_{\mathrm{m}}$ need not be very precise at all.

\subsection{PSF reconstruction}

The long exposure PSF may be obtained from the long exposure optical transfer function (OTF) $B(\rho / \lambda)=\langle B(\rho / \lambda, t)\rangle$ by Fourier transform, and the OTF in turn may be estimated by way of the phase structure function $D_{\varphi}(\boldsymbol{x}, \boldsymbol{\rho})$. Adopting the near-field approximation and neglecting amplitude fluctuations on the assumption of weak or low-altitude turbulence, the OTF may be written

$B(\boldsymbol{\rho} / \lambda)=\int \mathrm{d} x P(\boldsymbol{x}) P(\boldsymbol{x}+\boldsymbol{\rho})\left\langle\mathrm{e}^{i[\varphi(\boldsymbol{x}, t)-\varphi(\boldsymbol{x}+\boldsymbol{\rho}, t)]}\right\rangle$,

where $P$ is the pupil transmission function normalized to unit variance over the aperture. Invoking the central limit theorem, $\varphi(t)$ integrated along the line of sight becomes a normally distributed random variable with zero mean. The mean of a complex exponential with Gaussian statistics is $\langle\exp (i \varphi)\rangle=$ $\exp \left(-\frac{1}{2}\left\langle\varphi^{2}\right\rangle\right)$, and by the definition of the structure function

$D_{\varphi}(\boldsymbol{x}, \boldsymbol{\rho})=\left\langle|\varphi(\boldsymbol{x}, t)-\varphi(\boldsymbol{x}+\boldsymbol{\rho}, t)|^{2}\right\rangle$,

the statistical averaging of the exponential in (13) evaluates to $\exp \left[-\frac{1}{2} D_{\varphi}(\boldsymbol{x}, \boldsymbol{\rho})\right]$. Generally, the structure function of a partially corrected wavefront can be a function of both position $\boldsymbol{x}$ and correlation scale $\boldsymbol{\rho}$. In some instances, however, it may to a good approximation be replaced by its pupil average $\bar{D}_{\varphi}$

$\bar{D}_{\varphi}(\boldsymbol{\rho})=\frac{\int \mathrm{d} \boldsymbol{x} P(\boldsymbol{x}) P(\boldsymbol{x}+\boldsymbol{\rho}) D_{\varphi}(\boldsymbol{x}, \boldsymbol{\rho})}{\int \mathrm{d} \boldsymbol{x} P(\boldsymbol{x}) P(\boldsymbol{x}+\boldsymbol{\rho})}$.

Véran et al. (1997) discusses this to some extent. In the case when the phase errors are all tilts, this substitution will be perfectly valid, and the long-exposure OTF may presently be evaluated as

$$
\begin{aligned}
B(\boldsymbol{\rho} / \lambda) & =\exp \left[-\frac{1}{2} \bar{D}_{\varphi}(\boldsymbol{\rho})\right] \int \mathrm{d} x P(\boldsymbol{x}) P(\boldsymbol{x}+\boldsymbol{\rho}) \\
& =B_{\varphi} B_{\text {tel }},
\end{aligned}
$$

where the auto-correlation of the pupil function is the ideal telescope OTF, $B_{\text {tel }}$. Turning to the issue of tilt anisoplanatism, the sought after PSF is thus obtained from $B_{\varphi}$, which in turn requires the computation of the pupil-averaged phase structure function. Inserting (7) into (14) gives

$$
\begin{aligned}
D_{\varphi}(\boldsymbol{x}, \boldsymbol{\rho}, \boldsymbol{\theta}) & =\left\langle\left|\sum_{k=2}^{3} \gamma_{k}(\boldsymbol{\theta}, t)\left[Z_{k}\left(\frac{\boldsymbol{x}}{R}\right)-Z_{k}\left(\frac{\boldsymbol{x}+\boldsymbol{\rho}}{R}\right)\right]\right|^{2}\right\rangle \\
& =\sum_{k=2}^{3} \sum_{l=2}^{3} C_{\gamma}^{k l}(\boldsymbol{\theta}) U_{k l}(\boldsymbol{x} / R, \boldsymbol{\rho} / R),
\end{aligned}
$$

where

$U_{k l}(\boldsymbol{x}, \boldsymbol{\rho})=\left[Z_{k}(\boldsymbol{x})-Z_{k}(\boldsymbol{x}+\boldsymbol{\rho})\right]\left[Z_{l}(\boldsymbol{x})-Z_{l}(\boldsymbol{x}+\boldsymbol{\rho})\right]$.

Remains now only to evaluate $\bar{U}(\rho / R)$, since by linearity

$\bar{D}_{\varphi}(\boldsymbol{\rho}, \boldsymbol{\theta})=\sum_{k=2}^{3} \sum_{l=2}^{3} C_{\gamma}^{k l}(\boldsymbol{\theta}) \bar{U}_{k l}(\boldsymbol{\rho} / R)$.

Tilt modes without a central obscuration easily integrate analytically over a circular pupil, producing the matrix

$\bar{U}(\boldsymbol{\rho} / R)=\frac{4}{R^{2}}\left[\begin{array}{cc}\rho_{x}^{2} & \rho_{x} \rho_{y} \\ \rho_{y} \rho_{x} & \rho_{y}^{2}\end{array}\right]$,

which finally yields the quadratic form for the pupil-averaged phase structure function

$\bar{D}_{\varphi}(\boldsymbol{\rho}, \boldsymbol{\theta})=4 R^{-2}\left[C_{\gamma}^{22} \rho_{x}^{2}+C_{\gamma}^{33} \rho_{y}^{2}+2 C_{\gamma}^{23} \rho_{x} \rho_{y}\right]$.

Hence, the atmospheric tilt error is seen from (17) to be described by a Gaussian PSF whose width and orientation are field-dependent. This represents the very simplest case of PSF reconstruction, and the only case where the result obtained by imposing the pupil-averaging yields an exact representation. Were the same procedure to be attempted with more complicated modes, it might become necessary to seek a simplified approximate representation, but for the case of tilt anisoplanatism it is feasible, as shown, to work with the exact representation. The PSF retrieval algorithm is then clear: (i) build estimate of $C_{\mathrm{s}}$ from the closed loop WFS signals $s$, (ii) transform into $C_{\gamma}$ using the relation (12) and an estimate of $C_{\mathrm{m}}$, (iii) form the structure function via (22) and (iv) compute the long exposure OTF as in (16) and finally the PSF by Fourier transform. The presence of a central obscuration by the secondary mirror will not affect the results significantly so long as the NGS patrol field is unvignetted and the DMs are able to produce quadratic modes over the full pupil. Actuators within the obscuration that have localized influence functions may produce singular modes, but this can be overcome by using a precomputed calibration on an internal source to interpolate unilluminated actuators when the loop is closed and to constrain the formation of quadratic modes.

\section{Sample numerical results}

\subsection{Simulation configuration}

Figures $2-4$ present the results of a single run and compare the results of the estimation algorithm with the "true" PSFs and Strehl ratios delivered by the Monte Carlo simulation. Figures 5-7 present various diagnostics of the algorithm performance as the NGS magnitudes and input noise covariances are 


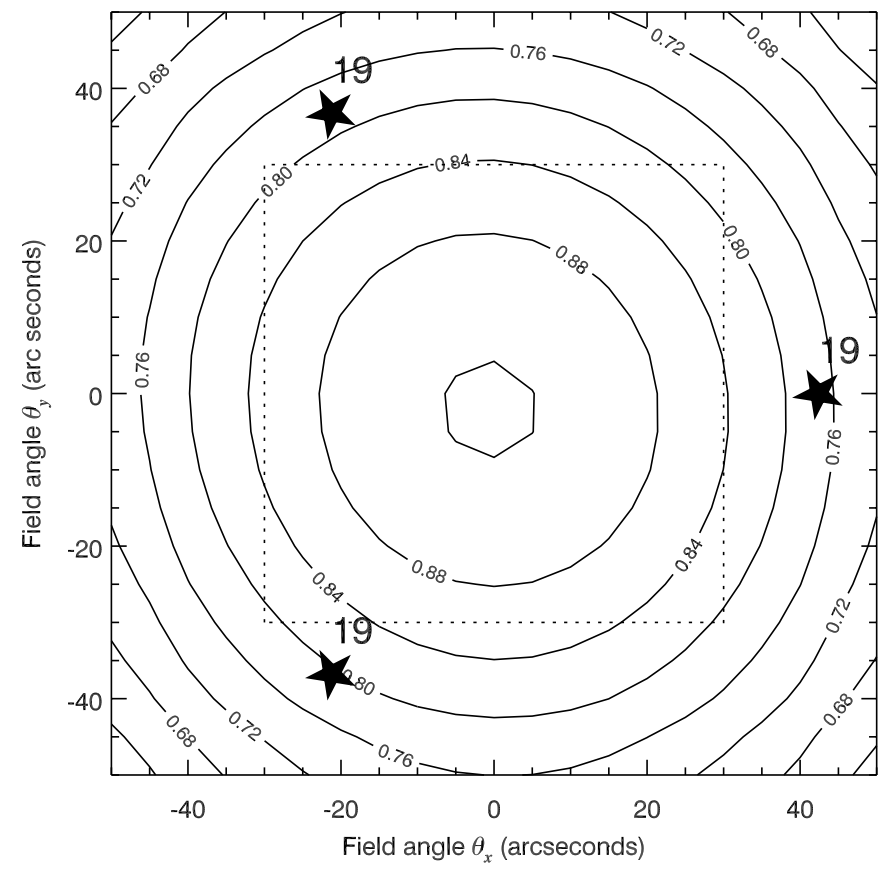

Fig. 2. $K$ band tilt anisoplanatism Strehl ratio over a 100 arcsec square field of view after a $30 \mathrm{~s}$ exposure at a sampling frequency of $500 \mathrm{~Hz}$. The three $m_{R}=19$ NGS are symmetrically distributed around the origin at a radial distance of $30^{\prime \prime}$.

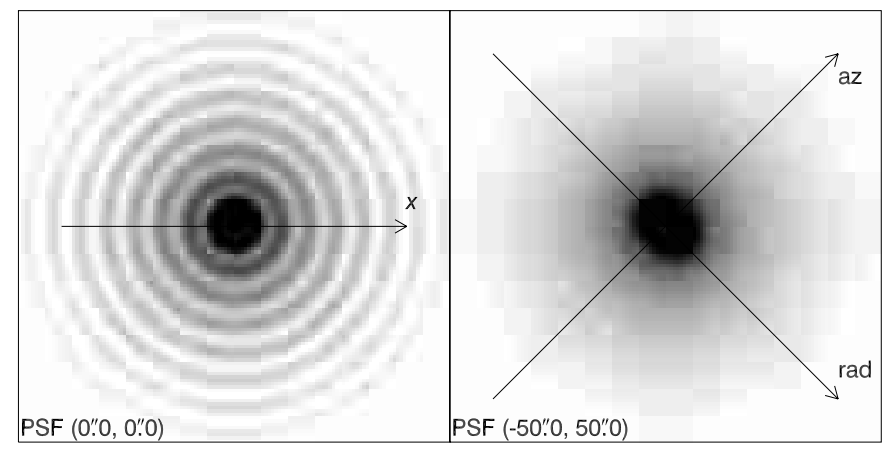

Fig. 3. Central (left) and peripheral (right) PSFs. The image has been chopped and stretched by a gamma correction of 0.1 .

varied. The numerical simulation used to generate closed loop data and evaluate performance is a trimmed down version of the Monte Carlo code used previously to simulate MCAO performance (Flicker et al. 2000). The turbulence in each layer of the model atmosphere was produced by the Zernikes (5), with time sequences of coefficients generated from Zernike temporal power spectral densities (PSDs). The PSDs were computed according to the formula derived by Roddier et al. (1993), modified for a finite outer scale $L_{0}=100 \mathrm{~m}$ and the integral normalized to the von Karman phase variance as calculated by Winker (1991). We adopted the turbulence profile tabulated in Table 1, which is a median 7-layer representation of the Cerro Pachon site survey (Vernin et al. 2000). This profile has an effective turbulence altitude $\bar{h}=3.9$, and an effective wind speed $\bar{v}_{x}=11.5 \mathrm{~m} \mathrm{~s}^{-1}$ along the $x$-axis. For testing the accuracy and robustness of the PSF reconstruction algorithm, and a nominal observation and system configuration was chosen as given in
Table 1. Median 7-layer Cerro Pachon turbulence profile, reporting the layer altitude $(h)$, the fractional turbulence strength $\left(C_{n}^{2} / \mu_{0}\right)$ and the wind speed along the $x$-axis $(v)$.

\begin{tabular}{lrrr}
\hline \hline LAYER & $h(\mathrm{~m})$ & $C_{n}^{2} / \mu_{0}$ & $v\left(\mathrm{~m} \mathrm{~s}^{-1}\right)$ \\
\hline $1 \ldots \ldots$ & 0 & 0.646 & 6.7 \\
$2 \ldots \ldots$ & 1800 & 0.078 & 8.3 \\
$3 \ldots \ldots$ & 3300 & 0.119 & 13.4 \\
$4 \ldots \ldots$ & 5800 & 0.035 & 25.6 \\
$5 \ldots \ldots$ & 7400 & 0.025 & 33.9 \\
$6 \ldots \ldots$ & 13100 & 0.080 & 22.2 \\
$7 \ldots \ldots$ & 15800 & 0.015 & 8.9 \\
\hline
\end{tabular}

Table 2. System and observation parameters: DM1 is the altitude conjugated deformable mirror, WFS the tip/tilt wavefront sensor, APD is the detector avalanche photodiodes and the reported quantum efficiency is over a $0.35 \mu \mathrm{m}$ bandwidth.

\begin{tabular}{lll}
\hline \hline System PARAMETER & & VALUE \\
\hline Telescope aperture diameter & $D$ & $4 \mathrm{~m}$ \\
DM1 conjugation range & $h_{\mathrm{M}}$ & $8 \mathrm{~km}$ \\
Air mass & $\mathrm{sec} \psi$ & 1 \\
Sky brightness (at $0.7 \mu \mathrm{m})$ & $m_{b}$ & $20.5 \mathrm{mag} /(\operatorname{arcsec})^{2}$ \\
Fried parameter (at $0.5 \mu \mathrm{m})$ & $r_{0}$ & $0.15 \mathrm{~m}$ \\
Imaging wavelength & $\lambda_{\text {im }}$ & $1.65 \mu \mathrm{m}$ \\
Sensing wavelength & $\lambda_{\text {sens }}$ & $0.7 \mu \mathrm{m}$ \\
WFS integration time & $\tau$ & $2 \mathrm{~ms}$ \\
APD angular subtense & $w$ & $1 \mathrm{arcsec}$ \\
Optical transmission & $\xi$ & 0.5 \\
Quantum efficiency & $\epsilon$ & 0.6 \\
\hline
\end{tabular}

Table 2. The symmetrical NGS asterism shown in Fig. 2, with three natural guide stars at a radial distance of $30^{\prime \prime}$ from the origin, was used throughout. Trials were run for asymmetric asterisms and widely varying NGS magnitudes within the range of operability of the null-mode system, and within this relatively small statistical sample the accuracy of the PSF reconstruction algorithm was found to be independent of the specific guide star configuration. Thus the symmetric asterism was chosen as the nominal configuration for the simulations presented here. The simulation did not consider additional tilt jitter from windshake, which will further reduce the field-averaged Strehl ratio and limiting NGS magnitude.

In the current design of the Gemini-South Adaptive Optics system, the NGS are picked up from a pre-focal collimated MCAO beam by three probe arms extracting $2^{\prime \prime} \times 2^{\prime \prime}$ beams (Stilburn 2001). The NGS beams are then imaged onto the detector area of the tip/tilt sensor, where a centroid measurement can be done. The noise on the spot centroid in terms of the one-axis rms measurement error was computed from the expression

$\sigma_{\mathrm{m}}=\frac{\theta_{B}}{S / N} \approx \frac{\lambda_{\text {sens }}}{r_{0}} \times \frac{0.587}{S / N}$

where $S / N$ is the NGS image signal-to-noise ratio, $r_{0}$ is the Fried parameter and $\theta_{B}$ is the effective spot size for the guide star image as given in (Welsh et al. 1995). For the case 

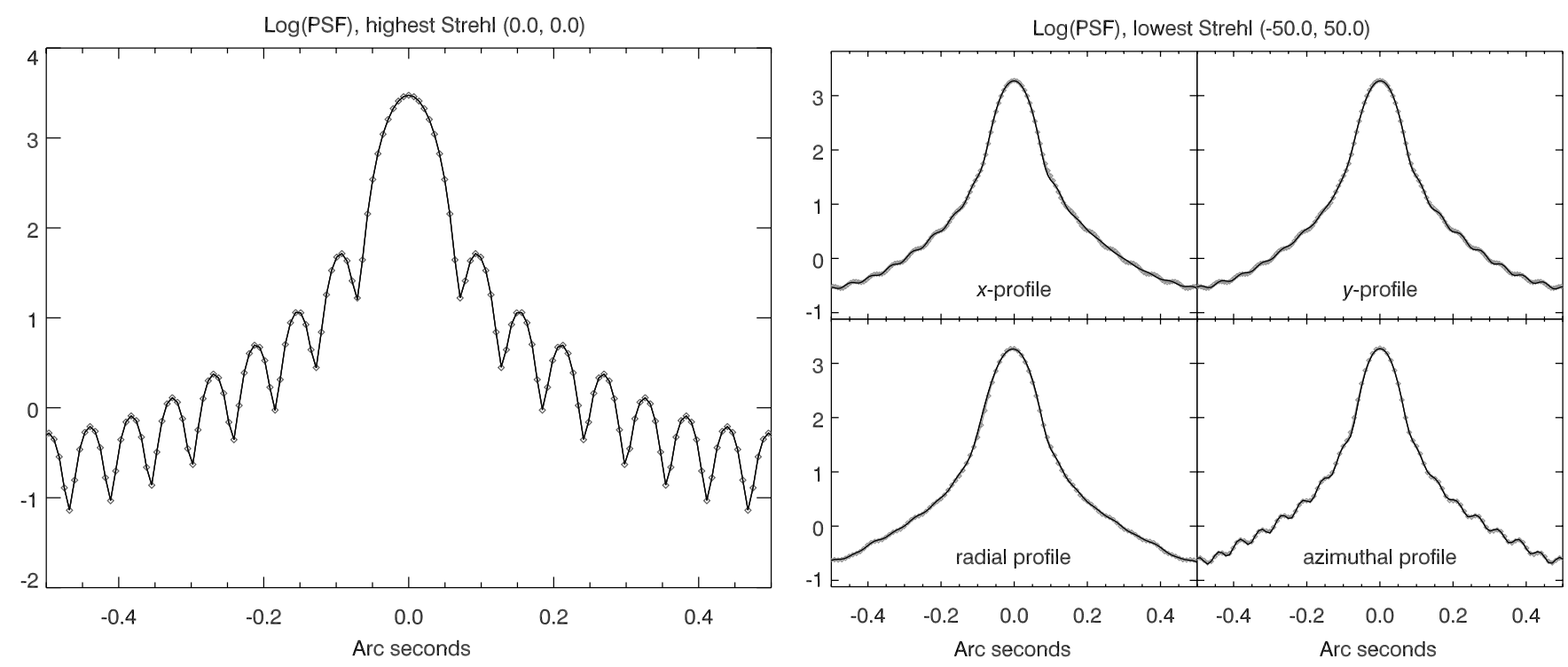

Fig. 4. Logarithm of the central (left, $x$-profile) and the peripheral (right, four different cross-sections) $K$ band PSF from a $30 \mathrm{~s}$ exposure with a 19th magnitude asterism. Solid - numerical simulation; diamonds - estimation. Radial and azimuthal profiles of the peripheral PSF as indicated by axes in Fig. 3.

of avalanche photodiode (APD) quadrant detectors, assuming zero dark current and read-out noise, the $S / N$ equation simplifies to $S / N=N_{\mathrm{p}} / \sqrt{N_{\mathrm{p}}+4 N_{\mathrm{b}}}$, where $N_{\mathrm{p}}$ is the total number of signal photodetection events and $N_{\mathrm{b}}$ is the number of background photodetection events per sensor pixel. Using standard radiometry formulae gives

$N_{\mathrm{p}}=z_{\mathrm{p}} \epsilon \xi A \tau 10^{-0.4\left[(\sec \psi-1) m_{\mathrm{a}}+m_{*}\right]}$,

$N_{\mathrm{b}}=z_{\mathrm{b}} \epsilon \xi A \tau w^{2}$,

where $m_{*}$ is the NGS magnitude, $\sec \psi$ the air mass, $m_{a}$ the atmospheric extinction in magnitudes per air mass, $\tau$ the integration time, $A$ the WFS pupil area, $w$ the angle subtended by a single APD, $\xi$ the end-to-end telescope beam transfer efficiency and $\epsilon$ is the APD quantum efficiency. The photometric zero point $z_{\mathrm{p}}$ is the intensity of a zero-magnitude guide star prior to attenuation by atmospheric extinction, and the background intensity $z_{\mathrm{b}}$ is reported per square arc second in the pupil plane of the telescope. Values for $z_{\mathrm{p}}$ and $z_{\mathrm{b}}$ were adopted from trial measurements done with the AO instrument Hokupa'a at the Gemini-North telescope (Rigaut 2002, private communication).

\subsection{PSF reconstruction results}

Null-mode compensation performance results of a single 30-s $K$-band exposure are shown in Figs. 2-4. The numerical simulation adopted the nominal system configuration given in Table 2, and the field of performance evaluation was $100^{\prime \prime} \times$ $100^{\prime \prime}$. Shown in Fig. 2 are iso-Strehl curves of the Strehl ratio resulting from tilt anisoplanatism for the symmetrical 19th magnitude NGS asterism as indicated by the stars; the averaged performance remains slightly asymmetric even after a 30-s exposure, due to the comparatively slow evolution of these low-order modes. In Fig. 3 two sample PSFs associated with the largest Strehl ratio (left), found at the center of the field covered in Fig. 2, and the lowest Strehl ratio found in the upper left corner of the field, are compared. Indicated in this image are also the axes along which the cross-sections in Fig. 4 were extracted. In Fig. 4 are plotted various cross-sections the two sample PSFs, extracted along the axes indicated in Fig. 3. It is seen that a $30 \mathrm{~s}$ exposure is sufficient for the algorithm to produce a very accurate estimate of the PSF even at the limiting magnitude. The $H$ and $K$ band system performance in terms of the Strehl ratio is plotted versus asterism magnitude in Fig. 5, where the three different cases give the performance on-axis, off-axis at the $\left(50^{\prime \prime}, 50^{\prime \prime}\right)$ corner of the square field, and as a field weighted average. The weights were chosen as the reciprocal of the multiplicity of the given field point for a radially symmetric turbulence compensation performance. The operability of the null-mode system may be gleaned from this plot, which corresponds well to previous investigations (including windshake tilt jitter) that inferred a $m_{R}=19$ limiting asterism magnitude, defined as the magnitude where the $H$ band Strehl ratio had dropped to 0.5 . Figures 6 and 7 plot the relative error in the Strehl ratio and the PSF for the PSF estimation algorithm, versus asterism magnitude, where the relative PSF error was computed as the root mean square (rms) of the estimation error divided by the rms value of the reference PSF produced by the numerical simulation. At the limiting magnitude $m_{R}=19$, the field-averaged relative error of the PSF estimation is less than $5 \%$ in both bands, which corresponds to a slightly smaller relative error in the Strehl ratio, $<1 \%$, which will be acceptable for most applications. It is notable, however, that even beyond the limiting magnitude and down to as faint as $m_{R}=21$, the estimation error does not get worse than $\sim 10 \%$, and even this may be improved upon somewhat by a longer exposure time. Whereas the relative error in the Strehl ratio provides a convenient and intuitive measure of the performance of the algorithm, the quantity relevant to photometry is the PSF rms error plotted in Fig. 7. One way to make a flux measurement on a 


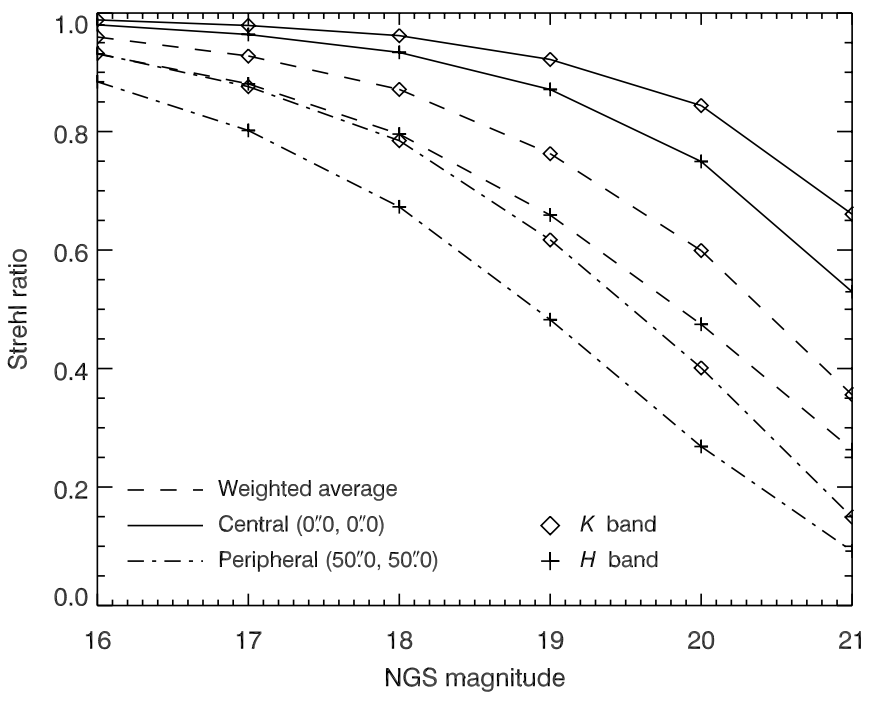

Fig. 5. $H$ (pluses) and $K$ (diamonds) band tilt anisoplanatism Strehl ratios versus asterism magnitude. Solid - central field point; dot-dashed - peripheral field point; dashed - field-weighted average.

star in a crowded field is to compute the full integral of a model PSF properly scaled to the star's brightness. Traditionally, this must be preceded by a PSF extraction sequence, and then the scaling of the model PSFs should be carried out simultaneously in order to minimize a global merit function. The error in flux is then directly related to the integral of the discrepancy between the model PSF and the star, and a measure of the flux uncertainty is the relative rms of this error, which is exactly what is plotted in Fig. 7. To investigate the impact of an error in the input noise covariance matrix $C_{\mathrm{m}}$, a set of matrices were computed for asterism magnitudes between $12 \leq m_{R} \leq 24$. The PSF reconstruction algorithm was then applied to simulations done for $16 \leq m_{R} \leq 22$, using this range of varying $C_{\mathrm{m}}$ as input to the algorithm. A local minimum as might have been expected at the correct noise magnitude was not observed for the relative reconstruction errors in the PSF and in the Strehl ratio. The results showed that the relative errors leveled out to the minimum level at a noise magnitude roughly equal to or slightly fainter than the correct magnitude, and no further degradation was observed at the bright end of the range of noise magnitudes. This suggests that the algorithm could in practice be made independent of $C_{\mathrm{m}}$ setting it to zero, or simply obviate the subtraction in (12). The data for Figs. 5-7 were averaged over 50 separate runs; whereas large asterism magnitudes converged faster, significant fluctuations in the individual results were observed toward the fainter end, which might not be entirely averaged after a sample of only 50 .

\section{Conclusions}

It was shown how the point spread function resulting from tilt anisoplanatism in a laser-guide-star-based multiconjugate adaptive optics system may be reconstructed with high accuracy over the entire field of view from closed loop wavefront sensor data. The reconstruction is robust with respect to noise and errors in the estimation of the noise covariance matrix, and

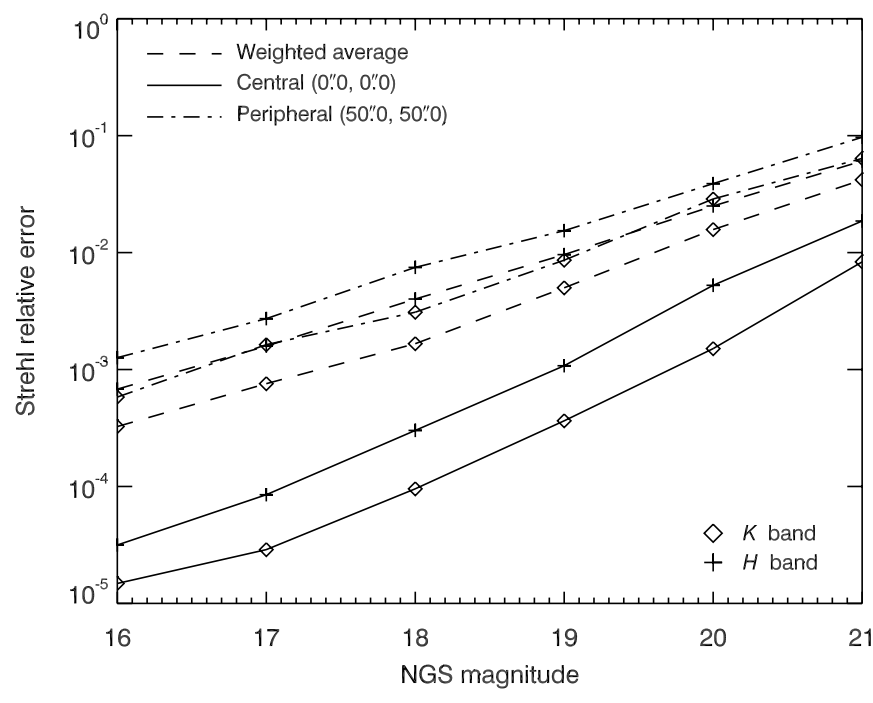

Fig. 6. Relative Strehl ratio estimation error in $H$ (pluses) and $K$ (diamonds) band. Solid - central field point; dot-dashed - peripheral field point; dashed - field-weighted average.

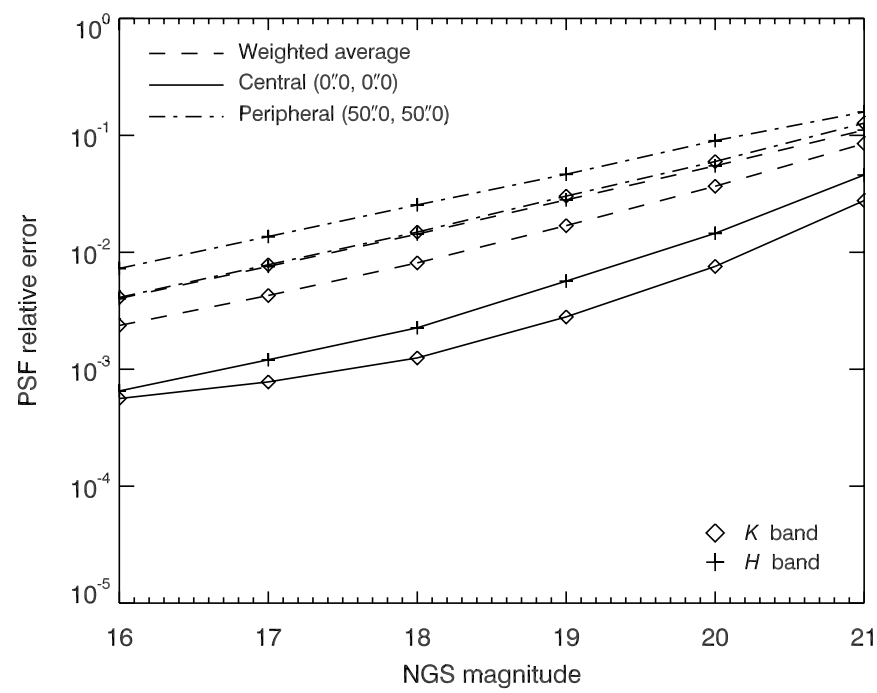

Fig. 7. Relative PSF estimation error in $H$ (pluses) and $K$ (diamonds) band. Solid - central field point; dot-dashed - peripheral field point; dashed - field-weighted average.

is shown to be accurate to within 5\% relative error at the $H$ band limiting magnitude, $m_{R}=19$. For longer wavelengths and at brighter asterism magnitudes, performance of the algorithm is much improved. Together with an algorithm for estimation the PSF variation due to partial correction of the high-order LGS system, a complete specification of the LGS MCAO PSF can be achieved, such as is indispensable for accurate photometry and deconvolution. A similar technique may be attempted for the high-order LGS system, but one can expect a lower degree of accuracy in this case as the success of this particular algorithm depends upon having precise knowledge of the modes over the field, which may not be possible for the LGS system with high enough accuracy. 
Acknowledgements. The author wishes to thank H.-G. Ludwig for contributing computing resources that rendered the computations of this paper possible within an acceptable time frame.

\section{References}

Beckers, J. 1988, in ESO Conf. on Very large telescopes and their instrumentation, ed. M.-H. Ulrich, 693

Ellerbroek, B. L. 1994, J. Opt. Soc. Am. A, 11, 783

Ellerbroek, B. L., \& Rigaut, F. 2001, J. Opt. Soc. Am. A, 18, 2539

Ellerbroek, B. L., \& Tyler, D. W. 1998, Publ. Astr. Soc. Pac., 110, 165

Flicker, R., \& Rigaut, F. 2001, in Beyond Conventional Adaptive Optics, ESO Conf. and Workshop Proc., 58, 377

Flicker, R., Rigaut, F., \& Ellerbroek, B. 2000, in Proc. SPIE, 4007, Adaptive Optical Systems Technology, 1032

Foy, R., Pique, J., Petit, A. D., et al. 2000, in Proc. SPIE, 4007, Adaptive Optical Systems Technology, 284

Fried, D. L. 1992, Fundamental limits in field widening for a multiple deformable mirror adaptive optics system, Tech. Rep. TR-1195, The Optical Sciences Company

Fried, D. L. 1995, in 1995 OSA Technical Digest Series, Adaptive Optics, ed. M. Cullum (Washington D.C.: Optical Society of America), 23, 363

Fugate, R. Q. 1994, J. Opt. Soc. Am. A, 11, 310

Gavel, D. T., \& Friedman, H. W. 1998, in Proc. SPIE, 3353, Adaptive Optical System Technologies, ed. D. Bonaccini, \& R. K. Tyson, 254
Hippler, S., Kasper, M. E., Feldt, M., et al. 2000, in Proc. SPIE, 4007, Adaptive Optical Systems Technology, ed. P. L. Wizinowich, 41

Le Louarn, M. 2001, in Beyond Conventional Adaptive Optics, ESO Conf. and Workshop Proc. 58, 217

Noll, R. J. 1976, J. Opt. Soc. Am., 66, 207

Parenti, R., \& Sasiela, R. 1994, J. Opt. Soc. Am. A, 11, 288

Pilkington, J. D. H., Thompson, L., \& Gardner, C. 1987, Nature, 330, 116

Racine, R., \& Ellerbroek, B. L. 1995, in Proc. SPIE, 2534, Adaptive Optical Systems and Applications, 248

Ragazzoni, R., Farinato, J., \& Marchetti, E. 2000, in Proc. SPIE, 4007, Adaptive Optical Systems Technology, ed. P. Wizinowich, 1076

Rigaut, F., \& Gendron, E. 1992, A\&A, 261, 677

Roddier, F., Northcott, M., Graves, J., McKenna, D., \& Roddier, D. 1993, J. Opt. Soc. Am. A, 10, 957

Stilburn, J. 2001, Appendix I, Internal report REV-AO-G0172, MCAO for Gemini-South: Preliminary Design Report, Gemini Observatory, 670 N. A’Ohoku P1., Hilo HI-96720, USA

Véran, J.-P., Rigaut, F., Maître, H., \& Rouan, D. 1997, in Proc. SPIE, 3126, Adaptive Optics and Applications, 81

Vernin, J., Agabi, A., Avila, R., et al. 2000, Gemini CP Site Characterization Report, Internal report RPT-AO-G0094, Gemini Observatory

Welsh, B. M., Ellerbroek, B. L., Roggemann, M. C., \& Pennington, T. L. 1995, in Proc. SPIE, 2534, 277

Winker, D. M. 1991, J. Opt. Soc. Am. A, 8, 1568 